Wir sehen somit, daß es im allgemeinen von Vorteil ist, zwischen Reihendrosselspulen und die zu schützenden Apparate nicht zu kurze Verbindungsleitungen mit möglichst niedrigem Wellenwiderstand zu legen; Schutzkondensatoren dagegen sind möglichst in der Nähe des zu schützenden Apparates anzuschließen.

Wir sehen auch ferner, daß, wenn die eben angegebenen Regeln befolgt werden, der Schutzwert der betrachteten Anordnungen ziemlich unabhängig von dem Wellenwiderstand der zu schützenden Apparate ist. Es ist dies von größter Wichtigkeit für den Schutz von Transformatoren, denn gerade der Wellenwiderstand von Transformatorenwicklungen schwankt innerhalb sehr weiter Grenzen.

\title{
Beitrag zur Berechnung der Achsen von elektrischen Maschinen.
}

\author{
Von
}

Erich Jasse, z. Zt. im Felde.

Bei der Durcharbeitung kleiner Gleichstrommaschinen zeigt es sich meist, daB der Raum für das aktive Ankereisen durch die Achse stark beeinträchtigt wird und man kommt leicht in die Versuchung, die Achse zugunsten des Ankerjoches zu schwächen. Dies geschieht um so leichter, als jeder $\mathrm{mm}$, den man gewinnt, bei der hohen Ankerinduktion großen Einfluß ausübt und es ist daher gerade bei diesen Maschinen angebracht, die richtige Wahl für die Abmessungen zu treffen, um nicht durch eine kräftige Achse unnötig am Ankerjoch zu verlieren und doch allen Anforderungen gerecht zu werden.

I. Die wirksamen Kräfte. Bei der Berechnung der Achse sind folgende Kräfte zu berücksichtigen, die von außen auf sie wirken:

a) Das Gewicht des Ankers und der Riemenzug; beide Kräfte sind für uns als konstant anzusehen, d. h. sie ändern sich nicht, wenn die Achse sich durchbiegt. Der Riemenzug soll dabei so angeordnet vorausgesetzt werden, daß er die Durchbiegung infolge des Gewichts vergrößert; dies ist der ungünstigste Fall. Beide Kräfte sollen daher zusammengefaßt und mit $P_{1}$ bezeichnet werden.

b) Der magnetische Zug $\mathrm{P}_{2}$; dieser hat keine Wirkung, solange der Anker genau zentrisch läuft, solange also der Luftspalt überall gleich groß ist; eine Exzentrizität nach irgend einer Seite wird durch diese Kraft vergrößert.

c) Die Fliehkraft $\mathrm{P}_{3}$; auch diese hat keine Wirkung, solange der Anker genau zentrisch läuft, nämlich solange der Schwerpunkt genau in der Umdrehungsachse liegt; auch hier versucht die Kraft, eine auftretende Exzentrizität zu vergrößern.

Es ist nun aber zu beachten, daß die Wirkungen von $P_{1}$ und $P_{3}$ nie gleichzeitig zu berücksichtigen sind. Denn $\mathrm{P}_{1}$ bringt eine zur Umgebung ruhende Durchbiegung hervor, und wenn $\mathrm{P}_{3}<\mathrm{P}_{1}$ ist, dann kann die Fliehkraft nicht in Erscheinung treten. Ist jedoch $\mathrm{P}_{3}>\mathrm{P}_{1}$, was entweder bei großer Drehzahl oder bei entgegengesetztem Riemenzug möglich ist und läuft der Schwerpunkt des Ankers um, so ist wegen der großen Drehzahl und der Massenträgheit des Ankers der Einfluß von $P_{1}$ ganz unwesentiich. $O b$ nun $P_{1}$ oder $P_{3}$ wirkt, der magnetische Zug versucht in jedem Falle die entstehende Ausbiegung der Achse zu vergrößern. Es kommt also entweder nur $\mathrm{P}_{1}$ und $\mathrm{P}_{2}$ oder $\mathrm{P}_{2}$ und $\mathrm{P}_{3}$ in Betracht.

Diesen Kräften wirkt nur die elastische Kraft $\mathrm{K}$ des Materials (die Federkraft) entgegen und diese ist daher genügend groß zu wählen, um keine unzulässige Durchbiegung eintreten zu lassen. 
Es bezeichne

$\delta$ den Luftspalt des Ankers, wenn dieser genau zentrisch läuft, so daß $\delta$ über den ganzen Umfang konstant ist,

$\varepsilon=\mathrm{x} \cdot \delta$ die Verschiebun ${ }^{r}$ der Ankermitte aus der Drehachse (die Exzentrizität).

Für den magnetischen Zuy auf einen Zylinder, der exzentrisch in einem Hohlzylinder liegt und an dessen Luftspalt eine konstante MMk wirkt, hat Sumec ${ }^{1}$ ) eine Formel abgeleitet, die mit unseren Zeichen wie folgt lautet

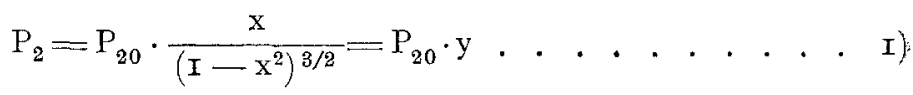

Die Fliehkraft eines Körpers ist proportional der Entfernung seines Schwerpunktes von der Drehachse; wir können also schreiben

$$
\mathrm{P}_{3}=\mathrm{P}_{30} \cdot \mathrm{x} \text {. . . . . . . . . . 2) }
$$

Eine gleiche Formel können wir auch für die Federkraft ansetzen, denn unterhalb der Proportionalitätsgrenze, und nur dieser Bereich kommt für uns in Betracht, ist die Kraft proportional der Durchbiegung, also hier der Exzentrizität. Es ist somit

$$
\mathrm{K}=\mathrm{K}_{0} \cdot \mathrm{x} \text {. . . . . . . . . . . . . . } 3
$$

a) Gewicht und magnetischer Zug.

Damit Gleichgewicht besteht, muß die algebraische Summe dieser Kräfte null sein, d. h. es muß sein

also

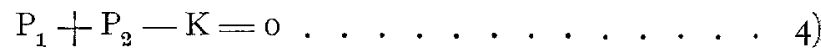

$$
\begin{aligned}
& \mathrm{P}_{1}+\mathrm{P}_{20} \frac{\mathrm{x}}{\left(\mathrm{I}-\mathrm{x}^{2}\right)^{3 / 2}}-\mathrm{K}_{0} \mathrm{x}=0 \quad \text {. . . . . . 4 4a) }
\end{aligned}
$$

Um uns eine klare Vorstellung von der Wirkung dieser Kräfte zu machen, wollen wir sie graphisch darstellen und zwar geschieht dies am besten in der in Abb. I gewählten Weise. Das Gewicht $\mathrm{P}_{1}$ ist eine Parallele zur x-achse (Kurve A); darüber

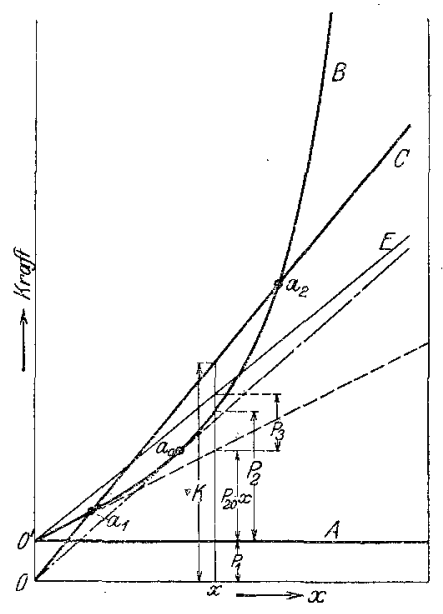

Abb. I. ist der magnetische Zug $\mathrm{P}_{2}$ nach Gl. I) aufgetragen (Kurve B). Ferner ist die Federkraft $\mathrm{K}$ aufgetragen, eine durch den Nullpunkt gehende Gerade (Kurve C). Diese letztere muß nun, damit ein Gleichgewichtszustand. überhaupt möglich ist, die Kurve B schneiden und zwar geschieht dies im allgemeinen in zwei Punkten $a_{1}$ und $\mathrm{a}_{2}$. Die durch Kurve $\mathrm{B}$ dargestellte Kraft $\left(\mathrm{P}_{1}+\mathrm{P}_{2}\right)$ versucht die Durchbringung zu vergrößern, sie wirkt nach rechts, während die durch Kurve $\mathrm{C}$ dargestellte Kraft $\mathrm{K}$ nach links wirkt. Damit nun der Gleichgewichtszustand stabil ist, muß die rückwirkende Kraft schneller ansteigen, als die ausbiegende und dies ist nur im Punkte $a_{1}$ der Fall. Im Punkte $a_{2}$ herrscht daher ein labiles Gleichgewicht. Verringern wir die Neigung der Geraden $\mathrm{C}$, also verkleinern wir die Federkraft, so rücken die beiden Punkte $a_{1}$ und $a_{2}$ mehr und mehr zusammen, bis sie schließlich zusammen-

fallen. Der dann gemeinsame Punkt $a_{0}$ stellt labiles Gleichgewicht dar und wir erkennen daher, daß stabiles Gleichgewicht nur in einern Punkte unterhalb $\mathrm{a}_{0}$ möglich ist. Als Bedingung für Stabilität findet sich somit die Beziehung.

$$
\left.\frac{\mathrm{dK}}{\mathrm{dx}}>\frac{\mathrm{d}\left(\mathrm{P}_{1}+\mathrm{P}_{2}\right)}{\mathrm{dx}} \ldots . . . . . . .5\right)
$$

I) J. K. Sumec, Berechnung des einseitigen magnetischen Zuges bei Exzentrizität; Zeitschr. f. Flektrot. rons. S. Er. 
Dieser Ausdruck hat ganz allgemeine Bedeutung, wenn wir auf der rechten Seite alle zur Vergrößerung der Ausbiegung beitragenden Kräfte zusammenfassen, auf der linken Seite alle rückwirkenden. Wir sahen vorhin, daß die Grenze der Stabilität erreicht war, wenn die Kurve der rückwirkenden Kräfte die der ausbiegenden berührte, d. h. wenn die Bedingung erfüllt ist

$$
\frac{\mathrm{dK}}{\mathrm{dx}}=\frac{\mathrm{d}\left(\mathrm{P}_{1}+\mathrm{P}_{2}\right)}{\mathrm{dx}}
$$

Voraussetzung für beide Beziehungen 5) und 6) ist natürlich, daß $K=P_{1}+P_{2}$ st (Gl. 3), daß also überhaupt Gleichgewicht vorhanden ist. Da nun die rückwirkende Kraft in unserem Falle eine durch den Nullpunkt gehende Gerade ist, so muß di. Tangente an die Kurve $B$ im Punkte $a_{0}$ ebenfalls durch den Nullpunkt gehen, $d . h$ es muß die Gleichung bestehen

$$
\frac{\mathrm{P}_{1}+\mathrm{P}_{2}}{\mathrm{x}^{3}}=\frac{\mathrm{d}\left(\mathrm{P}_{1}+\mathrm{P}_{2}\right)}{\mathrm{dx}}
$$

Hieraus findet sich mit Gl. I) die Bezeichnung

worin

$$
\left.\begin{array}{c}
\frac{P_{1}}{P_{20}}=x y^{\prime}-y=z \\
\frac{d y}{d x} ; \quad z=\frac{3 x^{3}}{\left(I-x^{2}\right)^{5 / 2}}
\end{array}\right\}
$$

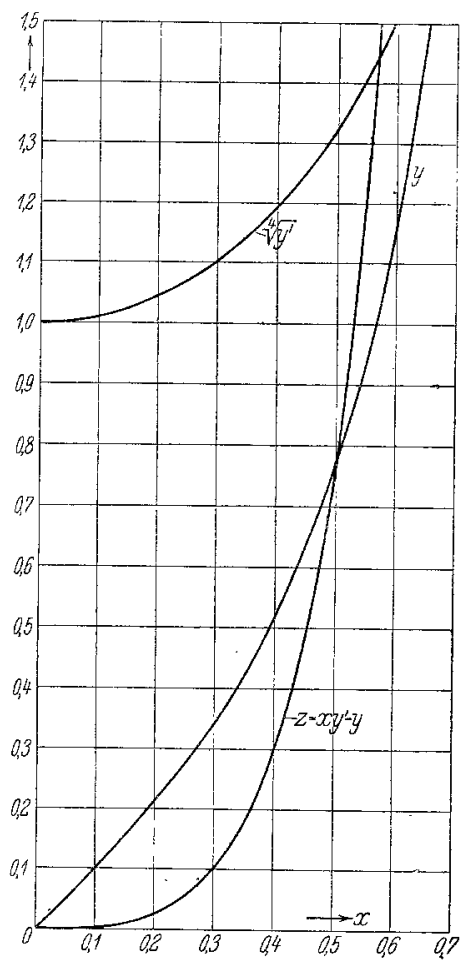

Abb. 2 .

In $\mathrm{Abb}, 2$ sind die Funktionen $\mathrm{y}$ und $\mathrm{z}$ über $\mathrm{x}$ aufgetragen. Da $\mathrm{P}_{1}$ und $\mathrm{P}_{20}$ bekannte Größen sind, so kann man $\mathrm{z}$ berechnen und aus der Kurve das zugehörige $\mathrm{x}$ entnehmen. Ferner findet sich aus Gl. 6)

$$
\mathrm{K}_{0}=\mathrm{P}_{20} \cdot \mathrm{y}^{\prime}=\mathrm{P}_{20} \frac{\mathrm{z}+\mathrm{y}}{\mathrm{x}} \cdot . .8 \text {. 8) }
$$

Die Federkonstante der Achse $\left(\mathrm{K}_{0}\right)$ und damit ihre Abmessungen können daher leicht berechnet werden.

Da Gl. 8) die Grenze für stabiles Gleichgewicht gibt, so wird man die rechte Seite mit einem Sicherheitsfaktor $\sigma$ multiplizieren, d. h. man wird die Federkraft $\sigma$ mal so groß wählen, wie sie für den Grenzzustand erforderlich ist. Die Größe $\sigma$ ist natürlich eine Erfahrungszahl.

$\beta)$ Fliehkraft und magnetischer Zug.

Die Gleichgewichtsbedingung heißt hier

oder

$$
\begin{aligned}
& \mathrm{P}_{2}+\mathrm{P}_{3}-\mathrm{K}=\mathrm{O} . . .9 .9 \text { ) } \\
& \left.\mathrm{P}_{20} \frac{\mathrm{x}}{\left(\mathrm{I}-\mathrm{x}^{2}\right)^{3 / 2}}+\mathrm{P}_{30} \mathrm{x}-\mathrm{K}_{0} \mathrm{x}=\mathrm{O} \cdot .9 \mathrm{9}\right)
\end{aligned}
$$

Wir sehen also schon aus dieser Gleichung, daß der Punkt $\mathrm{x}=0$ ein Gleichgewichtspunkt ist; alle drei Kurven gehen durch den Nullpunkt. In Abb. 3, S. 240 ist wieder $\mathrm{P}_{2}$ als ausbiegende Kraft (Kurve B) aufgetragen, während als rückwirkende Kraft $\mathrm{K}$ eingezeichnet (Kurve $\mathrm{C}$ ) und davon die Fliehkraft $P_{3}$ in Abzug gebracht ist (Kurve D). Wir sehen hier sofort, daß die Neigung der Geraden D -stärker sein muß, als die der Anfangstangente von Kurve B, damit ein stabiler Gleichgewichtszustand möglich ist und dann besteht dieser in der ideellen 
Drehachse $(\mathrm{x}=0)$. Die Grenze der Stabilität wird hier erreicht, wenn die Gerade D mit der Anfangstangente von Kurve $B$ zusammenfällt, d. h. wenn die Bedingung

$$
\mathrm{K}_{0}=\mathrm{P}_{20}+\mathrm{P}_{30} \text {. . . . . . . . . . . . Io) }
$$

erfüllt ist. Man muß also die Achsenstärke so wählen, daß $\mathrm{K}_{0}$ größer wird, als der durch Gl. Io) gegebene Wert, etwa $\sigma_{1}$ mal so groß, wenn $\sigma_{1}$ wieder ein Sicherheitsfaktor ist. Dann tritt aber eine Ausbiegung der Achse durch $\mathrm{P}_{2}$ und $\mathrm{P}_{3}$ gar nicht ein und daher kommt die Kraft $\mathrm{P}_{\mathbf{1}}$ wieder zur Wirkung.

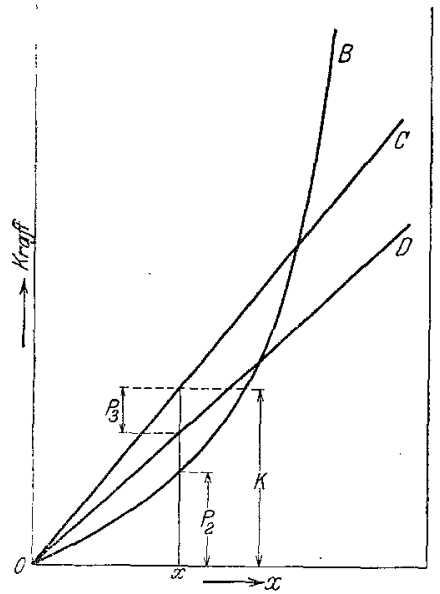

Abb. 3 . In Abb. I ist nun vom Punkte $O^{\prime}$ aus eine Gerade $E$ so aufgetragen, daß ihr Abstand (senkrecht zur $\mathrm{x}$-Achse gerechnet) von der Anfangstangente von $\mathrm{B}$ gleich $\mathrm{P}_{3}$ ist; die Ordinate dieser Geraden hat also den Wert $\mathrm{P}_{1}+\mathrm{P}_{20} \mathrm{x}+\mathrm{P}_{3}$. Um nun unter allen Umständen einen stabilen Lauf der Achse zu erhalten, ergibt sich die Bedingung, daß die Gerade $\mathrm{C}$ sowohl die Kurve B, als auch die Gerade $\mathrm{E}$ schneiden muß. Wenn wir nun die erforderliche Federkonstante der Achse bestimmen wollen, so müssen wir zuerst untersuchen, ob GI. 8) oder Gl. Io) den größeren Wert gibt und dann diesen mit unseren Sicherheitsfaktor multiplizieren.

2. Bestimmung der Kräfte aus den Abmessungen. Die Achse ist als Träger auf zwei Stützen anzusehen mit einer inneren Einzellast $\mathrm{P}^{\prime}$ und einer äußeren $\mathrm{P}^{\prime \prime}$. Die letztere stellt den Riemenzug dar, während die erstere $\mathrm{P}^{\prime}$ das Gewicht des Ankers, den magnetischen Zug und die Fliehkraft zusammenfaßt (siehe Abb. 4). Wir wollen hier jedoch unter $\mathrm{P}^{\prime}$ nur das Gewicht verstehen. Dann soll $\mathrm{P}_{1}$ diejenige Kraft sein, die in der Entfernung a vom linken Lager (an der Angriffstelle von $\mathrm{P}^{\prime}$ ) dieselbe Durchbiegung erzeugt, wie $\mathrm{P}^{\prime}$ und $\mathrm{P}^{\prime \prime}$ zusammen und wir erhalten

$$
\mathrm{P}_{1}=\mathrm{P}^{\prime}+\frac{\mathrm{c}}{\mathrm{b}}\left(\mathrm{I}+\frac{\mathrm{b}}{2 \mathrm{a}}\right) \mathrm{P}^{\prime \prime}
$$

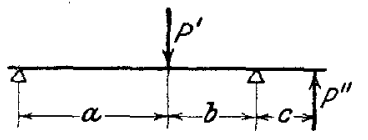

Abb. 4 .

Die Ableitung dieser Formel dürte sich an dieser Stelle erübrigen; mit Hilfe der elastischen Linie kann man den Ausdruck nach bekannten Regeln der Festigkeitslehre leicht erhalten. Für das Ankergewicht wollen wir die Gleichung ansetzen

$$
\mathrm{P}^{\prime}=\frac{\pi}{4} \mathrm{~d}^{2} \mathrm{l} \gamma \mathrm{g} \quad \text {. . . . . . . . . . . . . . . . . . }
$$

worin $\mathrm{d}$ und 1 die Hauptankerabmessungen (Durchmesser und Länge) sind und $\gamma$ ein mittleres spezifisches Gewicht darsteilt, wodurch Eisen, Kupfer und Lufträume berück. sichtigt sind ( $\mathrm{g}$ ist die Beschleunigung der Schwere). Es sei hier übrigens bemerkt, daß im absoluten elektromagnetischen Maßsystem gerechnet werden soll, so lange nichts anderes erwähnt ist. Den Riemenzug kann man im Mittel etwa gleich der dreifachen Umfangskraft setzen. Nimmt man den Durchmesser der Riemenscheibe gleich dem des Ankers, so wird

$$
\mathrm{P}^{\prime \prime}=3 \cdot 2 \frac{\mathrm{D}}{\mathrm{d}} \quad \text {. . . . . . . . . . . . . . . . . } \mathrm{I} 3 \text { ) }
$$

worin $\mathrm{D}$ das Drehmoment des Ankers bezeichnet und durch die Gleichung gegeben ist

$$
\left.\left.\mathrm{D}=\frac{\pi}{2} \kappa_{1} \mathrm{Bd}^{2} 1 \quad \text {. . . . . . . . . . . } \mathrm{I}_{4}\right)^{\mathrm{y}}\right)
$$

1) Diese Formel geht aus der auf S. 88 des Arch. f. Elektrot. für die Leistung abgeleiteten Gleichung ( $\mathrm{I}$ ) ohne weiteres hervor, da $\mathrm{I}_{\mathrm{a}}=\mathrm{D} \omega=\mathrm{D} 2 \pi \mathrm{n}$ ist. 
Hierin bedeutet:

$\alpha$ die relative Polüberdeckung, d. h. das Verhältnis des Polbogens zur Polteilung, $B_{1}$ die Induktion im Luftspalt,

S die Strombelastung des Ankers, d. h. seine Durchflutung bezogen auf die Einheit des Umfanges.

Man erhäIt somit für $P_{1}$ die Gleichung

$$
\left.\mathrm{P}_{1}=\frac{\pi}{4} \mathrm{~d}^{2} 1\left[\mathrm{~g} \gamma+\mathrm{I} 2 \frac{\mathrm{c}}{\mathrm{b}}\left(\mathrm{I}+\frac{\mathrm{b}}{2 \mathrm{a}}\right) \times \mathrm{B}_{1} \frac{\mathrm{S}}{\mathrm{d}}\right] \ldots . . . . \mathrm{I} 5\right)
$$

Die durch Gl. I) definierte Größe $\mathrm{P}_{20}$ ist, wie aus der Ableitung von Sumec hervorgeht, durch die Gleichung gegeben

$$
\left.\mathrm{P}_{20}=\frac{\mathrm{I}}{8 \pi} \mathrm{B}_{1}^{2} \cdot \pi \mathrm{d} 1 \alpha \ldots . . . . . . \mathrm{I} 6\right)
$$

Für die Größe $z$ in Gl. 7) ergibt sich daher der Ausdruck

$$
\left.z=\frac{P_{1}}{P_{20}}=\frac{2 \pi}{B_{1}^{2} \alpha} \cdot d\left[g \gamma+I 2 \frac{c}{b}\left(I+\frac{b}{2 a}\right) \alpha B_{1} \frac{S}{d}\right] . . . . . \quad I 7\right)
$$

Die Fliehkraft ist bekanntlich durch die Formel gegeben

$$
\mathrm{P}_{\mathbf{3}}=\mathrm{m} \omega^{2} \varepsilon
$$

wenn $\mathrm{m}$ die Masse und $\omega$ die Winkelgeschwindigkeit ist. Daher wird

$$
\left.\mathrm{P}_{30}=\frac{\pi}{4} \mathrm{~d}^{2} \mathrm{l} \gamma \omega^{2} \delta \quad \text {. . . . . . . . . . . . . . . } \mathrm{x} 8\right)
$$

Nun fehlt uns noch die Federkraft der Achse. Nach den Lehren der Festigkeit ist. diese für einen Träger auf zwei Stützen mit Einzellast durch die Formel gegeben ${ }^{1}$ )

$$
K=\frac{E J}{a^{2} b^{2}} 3(a+b) \varepsilon
$$

worin E der Elastizitätsmodul, J das Trägheitmoment des Trägerquerschnitts ist. Für einen kreisförmigen Querschnitt vom' Durchmesser $d_{1}$ ist

und daher erhalten wir

$$
\mathrm{J}=\frac{\pi}{64} \mathrm{~d}_{1}^{4}
$$

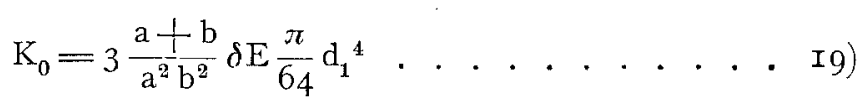

Wir hatten vorhin festgestellt, daß wir die Grenzwerte von $\mathrm{K}_{0}$ nach Gl. 8) und Gl. IO) zunächst auf ihre Größe zueinander untersuchen müssen. Durch Division der rechten Seiten erhalten wir mit Gl. I6) und I8) den Faktor

$$
\mathrm{f}==\frac{\mathrm{I}}{\mathrm{y}^{\prime}}\left(\mathrm{I}+\frac{\mathrm{P}_{30}}{\mathrm{P}_{20}}\right)=\frac{\mathrm{I}}{\mathrm{y}^{\prime}}\left[\mathrm{I}+2 \pi \frac{\gamma}{\alpha} \frac{\omega^{2}}{\mathrm{~B}_{\mathrm{l}}^{2}} \delta \mathrm{d}\right]
$$

Hierin ist $y^{\prime}$ durch Gl. 7) bestimmt und aus Abb. 2 mittels einer kleinen Zwischenrechnung leicht zu entnehmen. Solange nun $\mathrm{f}<\mathrm{I}$ ist, haben wir $\mathrm{K}_{0}$ aus Gl. 8 zu berechnen; ist jedoch $\mathrm{f}>\mathrm{I}$, so ist Gl. Io) zu benutzen. Wichtig ist hierbei der Fall $\mathrm{f}=\mathrm{I}$ und es empfiehlt sich, für eine zu entwerfende Maschine die diesem Grenzwert

1) Siehe z. B. "Des Ingenieurs Taschenbuch", Hütte, I9. Aufl. 1905, Bd. I S. 404, Fall 3. 
für den Einfluß der Fliehkraft entsprechende sekundliche Drehzahl $n_{0}=\frac{\omega_{0}}{2 \pi}$ aufzusuchen. Nach geringer Umformung erhält man mit Einführung von $\mathrm{n}_{0}$ den Ausdruok

$$
f=\frac{I}{y^{\prime}}\left[I+\left(y^{\prime}-I\right)\left(\frac{n}{n_{0}}\right)^{2}\right]
$$

der den Einfluß der Drehzahl besser zeigt.

Unsere beiden Bedingungsgleichungen 8) und Io) können wir nun auch vereinigen, indem wir schreiben

$$
\left.\mathrm{K}_{0}=\mathrm{P}_{20} \mathrm{fy}^{\prime} \text {. . . . . . . . . . . . } 2 \mathrm{I}\right)
$$

und die Anweisung geben, daß für Drehzahlen $n<n_{0}$ der Faktor $f=\mathbf{I}$ zu setzen ist.

3. Zahlenbeispiel. Es soll hier zunächst über den Durchmesser des Ankers noch keine Entscheidung getroffen werden, um die Abhängigkeit der Achsenstärke vom Ankerdurchmesser klarer erkennen zu können. Dies ist von Wert, wenn man eine Reihe von Maschinen steigender Größe zu entwerfen hat. Bei diesen ist im allgemeinen die Konstruktion grundsätzlich dieselbe, die meisten Abmessungen ändern sich angenähert proportional dem Durchmesser des Ankerș. Wir werden etwa setzen können:

$$
\mathrm{I}=0,8 \mathrm{~d} ; \mathrm{a}=\mathrm{I}, 8 \mathrm{~d} ; \mathrm{b}=\mathrm{I}, 5 \mathrm{~d} ; \mathrm{c}=0,67 \mathrm{~d} ; \delta=0,0 \mathrm{Id} \text {. }
$$

Ferner soll gewählt werden:

$$
\gamma=7 ; \alpha=0,67 ; \mathrm{B}_{1}=6000 ; \mathrm{S}=\mathrm{I}, 4 \mathrm{~d} ; \mathrm{E}=2,2 \cdot \mathrm{I0} \cdot 98 \mathrm{I} \text {. }
$$

Die Größe $\frac{\mathrm{S}}{\mathrm{d}}$ kann man, wenigstens bei kleinen Maschinen, als Konstante ansehen, folglich ist der Klammerausdruck von Gl. I5 bei Maschinen gleicher Konstruktion unabhängig von den Ankerabmessungen. Hiermit erhalten wir

$$
\begin{gathered}
\mathrm{P}_{1}=\frac{\pi}{4} \mathrm{~d}^{2} \cdot 0,8 \mathrm{~d}\left(98 \mathrm{I} \cdot 7+\mathrm{I} 2 \cdot \frac{\mathrm{0}, 67}{\mathrm{I}, 5} \cdot \frac{5, \mathrm{I}}{3,6} \cdot 0,67 \cdot 6000 \cdot \mathrm{I}, 4\right)=3,08 \cdot \mathrm{IO}^{4} \mathrm{~d}^{3}, \\
\mathrm{P}_{20}=\frac{\mathrm{I}}{8 \pi} 6000^{2} \cdot \pi \mathrm{d} \cdot 0,8 \mathrm{~d} \cdot 0,67=2,4 \cdot \mathrm{IO}^{6} \mathrm{~d}^{2},
\end{gathered}
$$

also wird

$$
\mathrm{z}=\frac{\mathrm{P}_{1}}{\mathrm{P}_{20}}=\frac{3,08 \cdot \mathrm{IO}^{4}}{2,4 \cdot \mathrm{IO} 0^{6}} \mathrm{~d}=\mathrm{I}, 29 \cdot \mathrm{IO}^{-2} \mathrm{~d}
$$

Für die Federkraft ergibt sich der Faktor

$$
\mathrm{K}_{0}=3 \cdot \frac{\mathrm{I}, 8 \mathrm{~d}+\mathrm{I}, 5 \mathrm{~d}}{\mathrm{I}, 8^{2} \mathrm{~d}^{2} \cdot \mathrm{I}, 5^{2} \mathrm{~d}^{2}} \cdot 0,0 \mathrm{Id} \cdot 2,2 \cdot \mathrm{IO}^{9} \cdot 98 \mathrm{I} \cdot \frac{\pi}{64} \mathrm{~d}_{1}^{4}=\mathrm{I}, 44 \cdot \mathrm{IO}^{9} \frac{\mathrm{d}_{1}^{4}}{\mathrm{~d}^{2}}
$$

folglich findet sich aus Gl. 8)

$$
\mathrm{I}, 44 \cdot \mathrm{IO}^{9} \frac{\mathrm{d}_{1}^{4}}{\mathrm{~d}^{2}}=2,4 \cdot \mathrm{IO}^{6} \mathrm{~d}^{2} \cdot \mathrm{y}^{\prime}
$$

oder

$$
\frac{\mathrm{d}_{1}}{\mathrm{~d}}=0,202 \sqrt[4]{\mathrm{y}^{\prime}}
$$

Wie man sich leicht überzeugt, ändert sich $\sqrt[4]{y^{\prime}}$ nur wenig mit dem Durchmesser; dehar ist $\frac{d_{1}}{d}$ ein nahezu konstanter Wert. Zur bequemen Berechnung ist der Wert $\sqrt[4]{y^{\prime}}$ in Abb. 2 ebenfalls in einer Kurve aufgetragen.

Für den Faktor $\mathrm{f}$ in Gl. 20) erhalten wir

$$
\mathrm{f}=\frac{\mathrm{I}}{\mathrm{y}^{\prime}}\left[\mathrm{I}+2 \pi \frac{7}{0,67} \cdot\left(\frac{2 \pi \mathrm{n}}{6000}\right)^{2} \cdot 0,0 \mathrm{Id} \cdot \mathrm{d}\right]=\frac{\mathrm{I}}{\mathrm{y}^{\prime}}\left(\mathrm{I}+0,724 \cdot 10^{-6} \mathrm{n}^{2} \mathrm{~d}^{2}\right)
$$


und daher wird der Grenzwert $\mathrm{n}_{0}$ für $\mathrm{f}=\mathrm{I}$

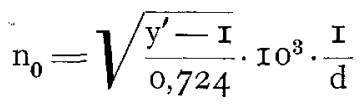

Die Ausrechnung ergibt für die drei Durchmesser $d=I 0,20$ und $30 \mathrm{~cm}$ die folgenden Zahlenwerte:

\begin{tabular}{c|c|c|c}
\hline $\mathrm{d}$ & I0 & 20 & 30 \\
\hline $\mathrm{z}$ & 0,129 & 0,258 & 0,387 \\
$\mathrm{x}$ & 0,320 & 0,387 & 0,426 \\
$\mathrm{y}^{\prime}$ & $\mathrm{r}, 570$ & $\mathrm{I}, 93 \mathrm{I}$ & $\mathbf{2 , 2 5 4}$ \\
\hline $\mathrm{n}_{0}$ & 89 & 57 & 44 \\
\hline $\mathrm{d}_{1}$ & 2,26 & 4,76 & 7,45 \\
$\mathrm{~d}_{1} / \mathrm{d}$ & 0,226 & 0,238 & 0,248
\end{tabular}

Wählt man den oben erwähnten Sicherheitsfaktor $\sigma$ etwa gleich 5, so würden die berechneten Achsendurchmesser um etwa die Hälfte stärker zu machen sein, da ja $\sqrt[4]{5} \approx x, 5$ ist.

Soll die Maschine mit $30 \mathrm{~cm}$ Ankerdurchmesser etwa $3000 \mathrm{U} / \mathrm{Min}$ machen, so ist der Grenzwert schon überschritten und man erhält

$$
\mathrm{fy}^{\prime}=\mathrm{I}+0,724 \cdot \mathrm{ro}^{-6} \cdot 5 \mathrm{0}^{2} \cdot 3 \mathrm{O}^{2}=2,63 \text {. }
$$

Daher ist mit Beachtung von Gl. 2I)

also wird

$$
\frac{\mathrm{d}_{1}}{\mathrm{~d}}=0,202 \cdot \sqrt[4]{2,63}=0,257
$$

$$
\mathrm{d}_{1}=7,73 \mathrm{~cm}
$$

Der Einfluß der Fliehkraft ist also schon bei dieser geringen Überschreitung der Grenze deutlich erkennbar; allerdings kommt er praktisch noch nicht in Betracht, da wegen des erforderlichen Sicherheitsfaktors der Unterschied reichlich gedeckt ist.

Es wäre jetzt noch zu untersuchen, ob die so bestimmte Achse auch stark genug ist, gegenüber dem im rechten Lager auftretenden Moment des Riemenzuges genügend Sicherheit zu bieten. Diese Rechnung ist jedoch aus der Festigkeitslehre genügend bekannt, so daß ein näheres Eingehen darauf hier nicht nötig ist.

Zusammenfassung. Es wird gezeigt, in welcher Weise die auf die Achse wirkenden Kräfte (Gewicht, magnetischer Zug, Fliehkraft) bei der Berechnung in Betracht zu ziehen sind, um einen stabilen Gleichgewichtszustand zu erhalten und von welchem Einfluß sie auf die Bemessung der Achsenstärke sind. Bei der Durchrechnung eines Zahlenbeispiels ergibt sich dann, daß man die Achsenstärke proportional dem Ankerdurchmesser machen kann. 